\title{
The continuing allure of RNA
}

\author{
JONATHA M. GOTT \\ Center for RNA Molecular Biology, Case Western Reserve University, Cleveland, Ohio 44106, USA
}

I feel incredibly fortunate to have had a career that spans so much of the flowering of RNA biology. Looking back, despite the fact that my initial projects centered on DNA and proteins, I was drawn to the field early on, starting with my very first journal club on the newly discovered intervening sequences in adenovirus mRNAs. Ultimately, it was my fortuitous decision to review the nascent field of group I and group II introns for a classroom assignment that led to the wonderful collaboration between the Shub and Belfort labs that firmly rooted me in the RNA world. Then, as a post-doc in the Uhlenbeck lab, my initial journal club covered all 5 extant research articles on RNA editing, setting me on my future path. Space constraints preclude listing all of the stunning discoveries that have been made over the last 20 years, so instead I will briefly mention highlights in three general areas (new roles, new techniques, new structures) before focusing on developments within my immediate field.

\section{New roles}

The expansion of our understanding of the myriad roles played by RNA has been truly remarkable. This includes the identification and functional characterization of whole new classes of RNAs (both small and large "non-coding"), regulatory pathways, defense mechanisms, and genome rearrangements. Heading the list is the discovery and continuing dissection of the RNAi/miRNA pathways. Although the Ambros and Ruvkin groups had established in 1993 that lin4 and let7 RNAs regulated their respective target mRNAs in C. elegans, the floodgates were opened in 2000 with the discovery that let7 was conserved across a wide evolutionary spectrum. The elegant genetic and biochemical studies that followed provide textbook examples for the next generation of scientists. In contrast, the investigation of the functional significance of lncRNAs has barely begun, but promises to be just as interesting. One area ripe for exploration is the world of small peptides potentially encoded by polysome-associated "non-coding" RNAs; roles in signaling pathways both within and between cells seem likely. The unfolding of the CRISPR story was another revelation, both in terms of

Corresponding author: jmg13@case.edu

Article and publication date are at http://www.rnajournal.org/cgi/doi/10. $1261 /$ rna.050252.115. Freely available online through the RNA Open Access option. the underlying biology of adaptive immunity in prokaryotes and its rapid adaptation for genome engineering. Nature has been using RNAs to guide genome engineering much longer, of course, as the studies of RNA-guided genome rearrangements in ciliates by Landweber and others have shown.

\section{New techniques}

Most of the above advances were only made possible by the development of powerful new methods. Microarrays and tiling arrays made it possible to assess the expression of hundreds, even thousands of genes at once. These were followed by the development of next-generation DNA and RNA sequencing methods, leading to the explosion of discovery seen in recent years. Because of these techniques, we have a much deeper appreciation of the degree of alternative splicing, polyadenylation, and pervasive transcription, as well as huge catalogs of newly discovered non-coding RNAs. Extensions of these high-throughput methods have enabled the development of a range of clever techniques for examining genome-wide RNA-protein interactions and higherorder genome structure. And, as discussed below, modification-specific methods are now revealing the existence of an extensive epitranscriptome. Other key developments include ribosome profiling and advances in structure probing that allow the assessment of RNA structure and dynamics.

\section{New structures}

The incredible number of high-resolution RNA and RNP structures that have been solved has also greatly influenced our current understanding of RNA structure-function relationships. The elucidation of the structure of the ribosome was a seminal event, the culmination of decades of effort toward understanding the workings of the translational machinery. Structures of other ribozymes, including group I and group II introns, hammerhead, hairpin, and RNaseP, have also yielded important insights into RNA-based catalysis. It was personally gratifying to see that the basic structure of group I introns closely resembles the pipe cleaner and toilet paper roll model that Eric Christian and I had built at our

\footnotetext{
(c) 2015 Gott This article, published in $R N A$, is available under a Creative Commons License (Attribution-NonCommercial 4.0 International), as described at http://creativecommons.org/licenses/by-nc/4.0/.
} 
kitchen table. Riboswitches have provided another set of fascinating structures, offering a 3D perspective into the dynamics of transcriptional and translational regulation. Further insights have been gleaned from other RNP structures, too numerous to count, that are now available.

\section{RNA editing and modification}

It has been fascinating to watch the field's evolution over the past 20 years, but I will list only a few high points, leaving it to the main protagonists in each area to elaborate further.

\section{Changes at the nucleotide level}

The 1986 discovery of trypanosome RNA editing in Benne's lab set off a frenzy of experiments in the labs of Stuart, Simpson, Sollner-Webb, Hajduk, and others. The identification of guide RNAs by the Simpson group led to models made testable by the establishment of in vitro editing systems. By 1995, the pendulum was swinging away from the trans-esterification model, but the debate wasn't resolved until 1996, when the Stuart lab provided convincing evidence for a cleavage-ligation mechanism. The identification and characterization of the multitude of proteins, complexes, and subcomplexes that carry out editing-related activities has proven to be an arduous task, but great progress has been made, aided by the advent of RNAi knockdowns and the additional efforts of Aphasizhev, Göringer, Read, Koslowsky, Cruz-Reyes, Alfonzo, and others. Meanwhile, other editing mechanisms were also emerging. Elegant experiments by Kolakofsky's group established that nucleotide insertions into paramyxoviral RNAs occur via polymerase stuttering within a slippery homopolymer tract. Extensive editing of mitochondrial (mt) mRNAs in Physarum was first discovered in 1991 by Dennis Miller, who went on show that, surprisingly, mt rRNAs and tRNAs also contain non-encoded nucleotides. In collaboration with Ralf Bundschuh, we extended this work by the first use of deep sequencing to define all editing sites within an organelle (1301 single and 23 di-nucleotide insertions, $4 \mathrm{C}$ to $\mathrm{U}$ changes, and replacement of the $5^{\prime}$ nucleotide of 2 tRNAs in Physarum). Our development of efficient in vitro editing systems led to the unexpected findings that inserted nucleotides are added to the $3^{\prime}$ end of nascent RNA via yet another form of co-transcriptional editing, while distinct post-transcriptional mechanisms are responsible for the observed base changes. Essential cis-acting elements within the DNA template were localized to an $\sim 18$ bp region centered around C insertion sites and evidence suggests that DNA-associated co-factors are also required. In nucleotide replacement editing, studies by the Gray and Jackson groups have demonstrated that repair of mismatches at the $5^{\prime}$ end of mt tRNAs is a post-transcriptional process involving deletion of the encoded nucleotide(s) and re-synthesis templated by the $3^{\prime}$ side of the acceptor stem. Intriguingly, this type of $3^{\prime}$ to $5^{\prime}$ polymerase activity may not be confined to the correction of tRNA ends. Mitochondrial tRNAs containing $3^{\prime}$ truncations (due to processing of overlapping tRNAs) or mismatches can also be repaired. The centipede reaction likely relies on a RNA-dependent polymerase that uses the $5^{\prime}$ side of the stem as a template, but other $3^{\prime}$ editing activities appear to be template-independent, adding As and/or Cs. This suggests that these editing enzymes may be related to poly-A polymerases or CCA-adding enzymes, potentially involving sequential rearrangements of the active site similar to those observed by Weiner and colleagues.

\section{Base changes}

$C$ to $U$ : The identification in 1987 of a specific $C$ to $U$ change in the apoB mRNA by the Scott and Chan labs was the first example of base alterations in mRNAs. By 1995, in vitro systems had been established, sequence requirements largely defined, and the catalytic component, APOBEC-1, identified, largely through work in the laboratories of the authors of the inaugural article in RNA (Davidson, Innerarity, Scott, Smith, Driscoll, Teng, and Chan). Cloning of the apobec1 gene by Davidson's group in 1993 also paved the way for the identification of other APOBEC family members (APOBEC2 and APOBEC3s, and activation-induced deaminase, AID), which play a range of roles in innate and adaptive immunity. Other highlights include the identification and cloning of the apobec-1 complementation factor (ACF), the expansion of known targets, links to obesity and disease, and investigation of developmental and regulatory aspects of editing. The discovery of $\mathrm{C}$ to $\mathrm{U}$ changes in plant mitochondrial mRNAs by the Gray, Grienenberger, and Brennicke labs in 1989 and in chloroplasts by Kössel's group in 1991 opened up another frontier. Plant mitochondrial RNAs are heavily edited, with hundreds to thousands of sites identified in recent genome-wide studies, whereas plastid RNAs are generally edited to only a modest extent. Sequence contexts immediately suggested that a distinct editing mechanism was at play, and by 1995 information on the cis-elements and trans-factors was starting to emerge from a combination of in vivo, in vitro, and in organello experiments. The key breakthrough, however, was the finding that organelle editing sites are specified by pentatricopeptide repeat (PPR) proteins, each of which recognizes only one or a few individual sites. Excitingly, a "PPR code" has recently been deciphered, permitting the prediction (and re-programing) of RNA binding specificity. Characterized specificity factors belong to the PLS subgroup of PPR proteins having $\mathrm{C}$ terminal extensions (E domains), about half of which also contain a terminal DYW domain. DYW domains share features with cytidine deaminases, leading to speculation that they provide the elusive editing activity, but this remains to be shown. Additional proteins that affect editing at many sites (RIP/MORF, ORRM) have recently been identified. These general editing factors interact with PPR proteins and are thought to function in the context of a larger "editosome." Notably, this 
form of editing may not be confined to plants since, although rare, PPR-DYW proteins have now been identified in other species, including some with known $\mathrm{C}$ to $\mathrm{U}$ changes. A to I: The biological relevance of the A to G/I? changes in ion channel mRNAs reported by Seeberg's group in 1991 was immediately apparent. A requirement for double-stranded regions was quickly recognized, implicating Bass' "unwindase," which converted A to I in dsRNA. In 1995 the production of inosine in crude editing extracts was confirmed, intensifying the pursuit of ADAR proteins. Many labs, including those of Bass, Beal, Emeson, Jantsch, Keller, Maas, Nishikura, O'Connell, Reenan, Samuel, and Seeburg, contributed significantly to the biochemical, biological, and genetic characterization of the A to I mechanism, aided by its occurrence in experimentally tractable model organisms. A to I changes have been observed in all types of RNAs, including miRNAs, and are particularly prevalent in the nervous system. ADAR knockouts are lethal in mice and editing defects have been tied to a number of pathological states. More recently, genome-wide approaches have greatly expanded the list of known targets, most of which fall outside coding regions, and intimated the existence of other types of base changes. $\mathrm{U}$ to $\mathrm{C}$ changes occur in some plant lineages and base alterations of many types have been reported in dinoflagellate organelles, but these remain to be characterized mechanistically.

\section{RNA modifications}

Although snoRNPs were known to be involved in ribosome biogenesis, it wasn't until 1996-1997 that the roles of the box $\mathrm{C} / \mathrm{D}$ and $\mathrm{H} / \mathrm{ACA}$ snoRNAs in guiding rRNA methylation and pseudouridylation were established by the Kiss, Steitz, and Fournier groups. Characterization of individual targets, snoRNP architecture, assembly, trafficking, and mechanisms followed, augmented by structural and genomic data, and modifications were introduced at novel sites with interesting outcomes. Contributions to stress responses, imprinting, splicing, translation, stability, miRNA generation, cancer, and other disease states have also been implicated. The recent development of methods such as m6A-seq, m5C-RIP, and bisulfite-seq has facilitated the assessment of the global distribution of modified nucleotides, giving rise to the field of epitranscriptomics. Thousands of $\mathrm{m} 6 \mathrm{~A}, 5 \mathrm{MeC}$, and pseudouridines have been mapped, falling within both coding and non-coding transcripts. Remarkably, like modifications in DNA and proteins, many of these RNA modifications appear to be inducible and reversible, potentially adding another layer to the regulatory landscape.

\section{Future directions}

Answers beget more questions, of course, and many key issues remain. How do the various complexes and sub-complexes coordinate the hundreds of $U$ insertions and deletions found in kinetoplast mRNAs? Where does the information for Physarum editing lie (i.e., what cis-elements and trans-acting factors specify the site and identity of the non-encoded nucleotides?). Are the tRNA editing enzymes part of a covert RNA repair network and, if so, what other substrates do they act upon? What are plant editosomes composed of, and what contributes the deaminase activity? What roles do A to I and $\mathrm{C}$ to $\mathrm{U}$ changes play in regards to regulation, development, and disease? To what extent do other base replacements occur, are they functionally significant, and how are they produced? What are the functions of the epitranscriptome? Are there other modifications yet to be identified? What underlying mechanisms contribute to their distribution, timing, specificity, and regulation? Clearly, there's plenty to look forward to in the next 20 years! 

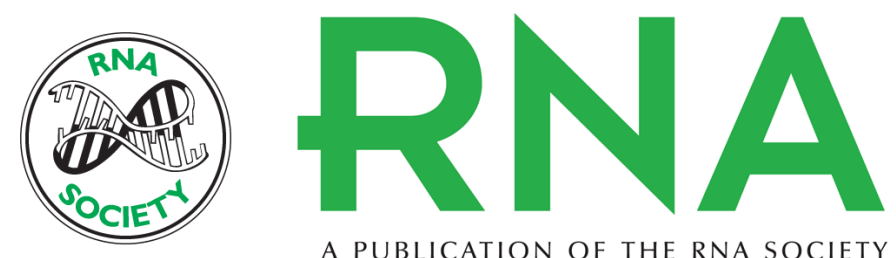

A PUBLICATION OF THE RNA SOCIETY

\section{The continuing allure of RNA}

Jonatha M. Gott

RNA 2015 21: 622-624

Open Access Freely available online through the RNA Open Access option.

Creative This article, published in $R N A$, is available under a Creative Commons License

Commons (Attribution-NonCommercial 4.0 International), as described at

License http://creativecommons.org/licenses/by-nc/4.0/.

Email Alerting Receive free email alerts when new articles cite this article - sign up in the box at the Service top right corner of the article or click here. 